\title{
Controllable Fe ion-anchored Graphene Heterostructures for Robust and Highly Thermal Conductive Cellulose Nanofiber Composites
}

\section{Bo Shan}

Nankai University

Yuzhu Xiong ( $\nabla$ xyzhu789@126.com )

Guizhou University https://orcid.org/0000-0001-6934-2672

\section{Research Article}

Keywords: Cellulose nanofiber, Fe ion-anchored graphene, Thermal conductivity, Interfacial interaction, Mechanical properties

Posted Date: July 12th, 2021

DOI: https://doi.org/10.21203/rs.3.rs-613510/v1

License: (c) This work is licensed under a Creative Commons Attribution 4.0 International License. Read Full License 


\section{Abstract}

Developing the polymer-based thermal interface materials (TIMs) is one of the most promising approaches to address heat accumulation along with the functionalization, integration, and miniaturization of modern electronics, while it is still a great challenge to balance the thermal conductivity and mechanical properties. In this article, Fe ion-anchored graphene (FeG) is successfully fabricated by a facile in situ Fe reduction of graphene oxide (GO) approach, and then cellulose nanofiber (CNF)/FeG composites are prepared by vacuum-assisted filtration. FeG exhibits excellent dispersion and exfoliation in CNF/FeG composites, due to the strong interfacial interaction between CNF and FeG, such as hydrogen bonds and "Fe-O" complex binding. Thus, CNF/FeG composite has the largely improved thermal conductivity up to $30.2 \mathrm{~W} / \mathrm{mK}$ at FeG content of $50 \mathrm{wt} \%$, which is substantially increased by $1160 \%$ in comparison with that of pure CNF. In addition, the mechanical performances of CNF/FeG-50 are unexpectedly simultaneously enhanced to $244 \mathrm{MPa}$ for tensile strength, $4.10 \%$ for elongation at break, and $9.5 \mathrm{GPa}$ for Young's modulus, outperforming pure CNF with increase of $137 \%, 33 \%$, and $121 \%$, respectively. This study provides a significant strategy for the design and construction of high thermal conductivity and high-performance polymeric TIMs in flexible and portable electronics.

\section{Introduction}

Heat accumulation causes serious efficiency and safety issues in rapidly growing fields, such as aerospace, 5G communication, electric vehicles, and sophisticated equipment manufacturing (Song et al. 2018; Ren et al. 2020; Chen et al. 2017). Central to the vital technical evolution is the development of high thermal conductive composites as thermal management materials (TMMs). To realize the high-thermal conductivity $(\mathrm{K}>10 \mathrm{~W} / \mathrm{mK})$ for composite materials, the design of 3D interconnection thermal conduction paths is regarded as the key factors, which is in favor of optimum utilization of finite filler. Han et al. reported a boron nitrides (BNNS)/epoxy composite based on nacre-mimetic 3D networks, which exhibits the high thermal conductivity of $6.07 \mathrm{~W} / \mathrm{mK}$ at $15 \mathrm{vol} \%$ BNNS loading, together with outstanding electrical resistivity and thermal stability (Han et al. 2019). Graphene, a superb thermally conducting filler, has been attracting the great interest of many researchers, due to its excellent thermal conductivity (5300 W/mK), high specific surface area and high aspect ratio (Song et al. 2018; Balandin et al. 2008; Duan et al. 2020). Wu et al. revealed a significant synergistic effect between the aligned graphene nanosheets (GNs) and 3D interconnected graphene foam (GF), which plays a key role in the formation of thermal percolation networks, leading to the thermal conductivity of $11.16 \mathrm{~W} / \mathrm{mK}$ at $10.27 \mathrm{vol} \%$ (Wu et al. 2019).

Apart from constructing artificial thermal conduction path through ice-templated self-assembly (Wu 2019; Schiffres 2013), liquid crystal induction (Meng et al. 2018), solvothermal synthesis (Xu 2010), melt migration limitation in block materials (Wu et al. 2017), etc., adding large amounts of well-dispersed fillers in the nanocomposites without excessive total mass or special processing methods is prone to multifunctional organic-inorganic hybrids (Xu et al. 2018). In most cases, the threshold is commonly about 30 vol.\% to realize the connection heat conduction path in composites (Song et al. 2018; Duan et al. 2020; Hu et al. 2017). Unfortunately, amounts of filler may be detrimental to the mechanical 
performances and processibility, and lead to a large equivalent thermal resistance caused by agglomeration (Schiffres et al. 2013; Xu et al. 2018; Yao et al. 2018; Guo et al. 2019; Ma et al. 2019).

Noticeably, previous studies has found that 1D cellulose nanofiber (CNF) possesses some ability to disperse graphene in water solution and finally be combined together into a strong composite by hydrogen bonding (Jiang et al. 2018), thus eliminating the complex chemical modification process and increasing the process efficiency (Zhao et al. 2020; Zhuo 2018; Malho 2012; Hamedi 2014; Xu 2018). Malho et al. first mixed CNF with multi-layer graphene, and then prepared a high-strength composite via intensely ultrasonic stripping (Malho 2012). The results demonstrated a simultaneous increase in Young's modulus (16.9 GPa), tensile strength (351 MPa), and toughness $\left(22.3 \mathrm{MJ} \mathrm{m}^{-3}\right)$ for the obtained composite, due to the unique hydrogen bonding, dipole effect, hydrophobic-hydrophobic interaction, and $\mathrm{CH}-\pi$ interaction between CNF and graphene (Hamedi 2014; Xu 2018; Chen 2018). However, with the increasing filler content, the mechanical properties can no longer keep up with high-strength applications, which is the common phenomenon in composites that lacks of specialized improvement (Malho 2012; Hamedi 2014; Xu 2018; Chen 2018). For example, Yang et al. took advantage of the synergistic performance of the 2D expandable graphite nanoplatelet (EG), 1D CNF, and flexible PEO. When the mass ratio of EG, CNF, and PEO reached to 95:5:3, the graphene-based composite displayed a significantly increased thermal conductivity and a decreased tensile strength about 63.3 MPa (Yang et al. 2019). Cui et al. successfully prepared hybrids based on nanodiamonds (NDs), graphene sheets (GSs) and CNF. The inplane thermal conductivity of the hybrid at a filler content of $10 \mathrm{wt} \%$ could reach to $14.35 \mathrm{~W} / \mathrm{mK}$, and the moderate tensile strength was $90 \mathrm{Mpa}$ (Cui et al. 2020). By contrast, balancing the thermal conductivity and mechanical properties under high loads is still a great challenge.

It is well known that the graphene oxides (GO) contains the ability to disperse into high-concentration water solution, owing to the electrostatic repulsion generated by the ionization of oxygen-containing functional groups that are introduced by edge oxidation of the graphene (Yuan 2013; Tian 2016; Dong 2018). Moreover, heat transport in graphene have been considered to rely on electron and phonon, therefore, the in-plane integrity lattice is regarded as the key factor to reduce phonon scattering and enhance the thermal conductivity. We propose that a non-covalent modification method focused on edge binding of reduced $\mathrm{GO}(\mathrm{rGO})$ is a feasible strategy to balance the thermal conductivity and mechanical properties. Wei et al. reported that the $\mathrm{sp}^{2}$ hybrid orbital lattice could be restored by the oxidationreduction reaction with the participation of Fe ions, but the oxygen-containing functional group at the edge could be well remained (Fan 2011). This controlled reduction of GO has achieved satisfactory results in terms of energy storage and adsorption separation. However, the role of edge-functionalized non-covalent modification graphene in thermal conductive composites has not been detailedly explored yet.

In this paper, Fe ion-anchored graphene ( $\mathrm{FeG}$ ) is successfully in situ constructed via the controllable Fereduction of $\mathrm{GO}$. FeG exhibits the high compatibility with $\mathrm{CNF}$, owing to the intense interaction between FeG and CNF (e.g., hydrogen bonds and "Fe-O" complex bonds). Under vacuum filtration, CNF/FeG composite possess the dense and compact structures with high in-plane alignment. As a consequence, 
the thermal conductivity of CNF/FeG-50 is greatly enhanced, due to the continuous thermal conduction path built by aligned FeG sheets. The mechanical performances of CNF/FeG-50 are also a lot higher than those of pure CNF, due to the superb reinforcing effect of FeG. The flexible CNF/FeG composites with both favorable thermal conductivity and mechanical properties open a potential application in microelectronics and advanced energy.

\section{Material And Methods}

\section{Materials}

The cellulose nanofiber (CNF) dispersion with a carboxyl content of approximately $1.08 \mathrm{~mol} \mathrm{~g}^{-1}$ was provided by CANFOR Co., Ltd. (Canada). Graphene oxides (GOs) and graphene nano-plates (GNPs) were supplied by ENN Graphene Technology Co., Ltd (China). The anhydrous ethanol, Fe powder and $\mathrm{HCl}$ were purchased from Tianjin Kemio Chemical Reagent Co., Ltd (China). The reagents were all used without further purification.

\section{Preparation of Fe ion-anchored graphene (FeG)}

Figure 1 schematically shows the preparation method for FeG (Fan 2011). First, $10 \mathrm{~g}$ of graphene oxide was weighed in a wide-mouth beaker and dispersed in $1 \mathrm{~L}$ of distilled water using a $600 \mathrm{~W}$ sonification. Secondly, $50 \mathrm{~g}$ of 300 mesh iron powder and $30 \mathrm{~mL}$ of $35 \mathrm{wt} . \% \mathrm{HCl}$ solution were added into the above dispersion to allow the redox reaction. After $24 \mathrm{~h}$ reaction, the product was washed several times with deionized water to neutralization. Lastly, FeG was obtained by freeze-drying for $48 \mathrm{~h}$

\section{Preparation of CNF/FeG composites}

The homogeneous mixture suspensions with different FeG contents $(0,10,30$, and 50 wt.\%) were prepared via mechanically stirring for $30 \mathrm{~min}$ and subsequent ultrasonic treatment for $20 \mathrm{~min}$.

Afterwards, the above suspension was vacuum-filtered via a hydrophilic ester filter membrane with a pore size of $0.22 \mu \mathrm{m}$ and diameter of $47 \mathrm{~mm}$. The CNF/FeG composites and pure CNF were taken off from the substrate membranes after air-drying overnight at room temperature.

\section{Characterizations}

Scanning electron microscope (SEM) images were obtained using a JSM-7500F microscope (JEOL, Japan) at an operating voltage of $5 \mathrm{kV}$. The X-ray diffraction (XRD) patterns were recorded on an X-ray diffractometer ( $\mathrm{D} / \mathrm{max} 2200$, Rigaku, Japan) in Cu Ka radiation $(\mathrm{k}=1.5406 \AA)$ at $40 \mathrm{kV}$, scanning from $5^{\circ}$ to $40^{\circ}$ at a speed of $4^{\circ} / \mathrm{min}$. After drying at $50^{\circ} \mathrm{C}$ for $6 \mathrm{~h}$, the chemical structures of hybrids were characterized through attenuated total reflectance Fourier-transform infrared (ATR-FTIR) spectroscopy (Nicolet iS50, U.S.A.) over the range of $4000-400 \mathrm{~cm}^{-1}$ and Raman spectroscopy (Renishaw Invia, U.K.) using a Ne-Ha laser. The "in-plane" mode thermal diffusion coefficient k was measured by the laser flash Netzsch LFA 467 at $25^{\circ} \mathrm{C}$. The thermal conductivity $(K)$ was then determined as $K=\rho \cdot k \cdot C_{p}$, where $\rho$ is the mass density measured by drainage method and $C_{P}$ is the specific heat obtained by differential scanning 
calorimetry (DSC). The tensile properties of the obtained composites were measured using an Instron tester (INSTRON 5865) at a loading speed of $0.5 \mathrm{~mm} / \mathrm{min}$.

\section{Discussion}

\section{Preparation and properties of FeG}

The preparation process for $\mathrm{FeG}$ is intuitively illustrated in Fig. 1a. During the reduction of GO powder to $\mathrm{FeG}$ at the presence of $\mathrm{Fe} / \mathrm{Fe}^{2+}$, the volume is largely expanded, which greatly enhances the specific surface area of $\mathrm{FeG}$ and interfacial interaction between CNF and FeG. The obtained FeG has good dispersion in CNF aqueous solution, as shown in Fig. 1C. A dense and continuous FeG coating is formed on the glass rod (Fig. 1d), which proves that the obtained FeG also has good self-assembly activity (Zhao 2017; Ramanathan 2008).

The Raman spectra of Go, FeG and GNP are comparatively shown in Fig. 2a. The peak intensity ratio $I_{D} / I_{G}$ of $\mathrm{FeG}$ is remarkably decreased from 1.14 to 0.86 in contrast with that of GO, which indicates an improved in-plane $\pi-\pi$ conjugated structure (Ferrari and Robertson 2000). Considering that the reduction process may lead to size reduction, the actual $I_{D} / I_{G}$ value may be even lower (Ferrari 2000). D- and Gbands of FeG shift to lower wavenumber after reduction, mainly due to the Fe- 0 charge transfer (Geng 2013). This implies that parts of the oxygen-containing functional groups preserved after the $24 \mathrm{~h}$ reduction are mainly centered on the edge of graphene (Jha 2017). Moreover, the symmetrical secondorder phonon scattering peak (i.e., 2D peak) of FeG located at $2700 \mathrm{~cm}^{-1}(532 \mathrm{~nm})$ can be decomposed into four peaks, which indicates that a certain amount of bilayer graphene exists among FeG (Ni 2008). The XRD diagrams (Fig. 2b) show that the strong diffraction peak traced at $26^{\circ}$ for GNP is corresponding to the plane lattice (002). However, the (002) plane diffraction peak of FeG is observed over a wide range of $21-26^{\circ}$, signifying a large amount of exfoliated graphene nanosheets (Cui 2011). Such a phenomenon takes place because $\mathrm{Fe} / \mathrm{Fe}^{2+}$ ions are largely intercalated between $\mathrm{GO}$ layers as the reaction proceeds (Geng 2013). From the Scherrer formula, the sharp shoulder peak at $21^{\circ}$ in FeG XRD curve is corresponding to the planar spacing of about $4.2 \AA$, which indicates the existence of 1-2 layer graphene after the reduction. The SEM image of FeG is displayed in Fig. 2c. FeG exhibits a thin layer structure with many wrinkles, which indicates the pretty exfoliation of FeG (Stankovich 2006; Park 2014).

FTIR spectra are used to determine the types of oxygen-containing functional groups on FeG (Fig. 3). In $\mathrm{GO}$, the broad and intense hydroxyl peak is situated at $3304 \mathrm{~cm}^{-1}$, and the asymmetrical and symmetrical stretching vibrations of $-\mathrm{CH}_{2}$ are located at 2925 and $2856 \mathrm{~cm}^{-1}$, respectively. The prominent peak at about $1750 \mathrm{~cm}^{-1}$ is corresponding to ester $\mathrm{C}=0$ stretching vibration. The characteristic peaks indexed to carboxy/carbonyl and aromatic $\mathrm{C}=\mathrm{C}$ stretching vibrations are observed at 1637 and $1539 \mathrm{~cm}^{-1}$, respectively. The peak existing at $1222 \mathrm{~cm}^{-1}$ is assigned to epoxy $\mathrm{C}-\mathrm{O}$ stretching vibration. The bands at 1150 and $1050 \mathrm{~cm}^{-1}$ are the respective stretching vibrations of the tertiary and primary alcohol C-O (Lu 2008). 
After reduction by $\mathrm{Fe} / \mathrm{Fe}^{2+}$, the hydroxyl band centered at the higher wavenumber $\left(3440 \mathrm{~cm}^{-1}\right)$ for $\mathrm{FeG}$ presents the observably decreased intensity, which illustrates the effective removal of oxygen-containing functional groups and the strong "Metal-Oxygen" (Fe-O) interaction (Scott 2013). Accordingly, the ester C $=0$ and epoxy $\mathrm{C}-\mathrm{O}$ bands are remarkably weakened with the reduction proceeding, because of the reaction between the epoxy groups and Lewis alkali metal ions (He 1998; Lerf 1998). Natheless, the carboxy $\mathrm{C}=\mathrm{O}$ and aromatic $\mathrm{C}=\mathrm{C}$ are largely augmented, indicating the restored $\pi-\pi$ conjugated structures. In addition, plenty of primary alcohol C-O bonds are converted to tertiary alcohol, possibly owing to the recovery of $\mathrm{C}-\mathrm{C}$ bonds. The results infer that some certain oxygen-containing functional groups remain after Fe-reduction, most of which exists in the form of carboxyl groups at the edge of FeG sheets.

\section{Morphology, structures and mechanical properties of CNF/FeG composites}

To explore the morphology and microstructures of CNF/FeG composites, cross-section SEM images of CNF/FeG-50 are depicted in Fig. $4 a$ and b. At such high FeG loading of 50 wt\%, CNF/FeG-50 displays a highly aligned and ordered layer-by-layer structure, which is essential to the high performance of $\mathrm{CNF} / \mathrm{FeG}$ composites. This aligned structure heavily depends on the good dispersion and exfoliation of FeG at the assistance of CNF/Fe ion. CNF with large number of hydrophilic groups (- $\mathrm{COOH}$ and $-\mathrm{OH}$ ) can be easily intercalated into FeG interlamination through the induction of Fe ions, which is profitable for the pretty dispersion and stability of FeG in CNF solution (Fig. 4d). As shown in Fig. 4c, the positive Fe ions introduced on FeG also play a pivotal role in assisting FeG exfoliation via the dynamic "Metal-O" bond interaction between Fe ions and $\mathrm{CNF}$ in terms of $\mathrm{CH}-\pi$ interaction, dipole interaction, hydrophobichydrophobic interaction, etc. The interaction forces can act as the gripper of CNF and FeG to more effectively strip FeG sheets under shear flow.

Figure $4 \mathrm{e}$ and $\mathrm{f}$ describe the FTIR spectra of pure CNF and CNF/FeG composites. It is visually found that the characteristic -OH peak slightly blue-shifts from $3252 \mathrm{~cm}^{-1}$ for pure CNF to $3322 \mathrm{~cm}^{-1}$ for CNF/FeG50 with the increasing FeG content (Fig. 4f), which illustrates the strong hydrogen binding between FeG and CNF. Similarly, $\mathrm{C}=\mathrm{O}$ and $\mathrm{C}-\mathrm{O}$ groups in carboxy moderately red-shifts with the addition of FeG content, due to a strong "Metal-Oxygen" complex bond between FeG and CNF (Fig. 4e) (Zhao 2017; Kemp 2017). In brief, the interfacial interaction between FeG and CNF in CNF/FeG composites is largely enhanced with the introduction of Fe ions, which is vital to the improvement of mechanical properties of CNF/FeG composites.

After the controllable reduction, the in-plane carbon lattice integrity of FeG is largely improved, so the stress load substantially increases in comparison with that of GO. Moreover, the complexation of Fe ions with oxygen-containing groups exerts a significant effect on the mechanical properties of CNF/FeG composites. Figure 5a-c show the tensile properties of pure CNF and CNF/FeG composites. It is obviously observed that pure CNF possesses the relatively poor mechanical poor mechanical performances, regrading strength of $103 \mathrm{MPa}$, elongation at break of 3.08\%, and Young's modulus of $4.3 \mathrm{GPa}$. All the 
characteristic mechanical properties of CNF/FeG composites are enhanced with the incremental FeG loadings. At a high FeG content of $50 \mathrm{wt} \%$, the exceptionally improved strength, elongation at break, and Young's modulus reach up to $244 \mathrm{MPa}, 4.10 \%$, and $9.5 \mathrm{GPa}$, respectively, which is substantially increased by $137 \%, 33 \%$, and $121 \%$, respectively, in contrary to those of pure CNF. This phenomenon is primely attributed to the strong interactions at CNF/FeG interfaces concerning hydrogen bond and "MetalOxygen" bridge binding, which has been confirmed by the analyses of Raman and FTIR spectra. Generally speaking, a handful of metal ions can be penetrated into or through the interior space of graphene layers, limited by the radius of metal ions, which results in the insufficient interfacial bridge binding (Zhang 2018; Park 2008; Gong 2017; Wan 2018; Shen and Feng 2018). However, abundant Fe ions are anchored on FeG sheets by " $\mathrm{Fe}-\mathrm{O}$ " complexation bonds during the controllable Fe reduction. The EDS line scanning spectrum (Fig. 5d) unveils the high Fe element fraction of 28.4 wt\% in CNF/FeG-50 composite. It is found in EDS mappings (Fig. 5e-f) that most Fe element is gathered at the FeG region, which manifests that $\mathrm{Fe}$ ions are firmly bonded on FeG sheet and not washed out in aqueous solution. The strongly anchored Fe ions on FeG can tightly link to CNF via "Metal-Oxygen" bonds, contributing to the reinforced mechanical properties of CNF/FeG composites.

\section{Analysis of thermal conductivity of CNF/FeG composites}

The in-plane thermal diffusivity and thermal conductivity of CNF/FeG are shown in Fig. 6a and c. It is visual that the in-plane thermal conductivity of pure CNF is merely $2.4 \mathrm{~W} / \mathrm{mK}$, in good agreement with previous reports (Shen 2018; Uetani 2016). The thermal conductivity of CNF/FeG is evidently enhanced with the introduction of FeG. When $10 \mathrm{wt} \% \mathrm{FeG}$ is added, the thermal conductivity of CNF/FeG composite reaches up to $9.3 \mathrm{~W} / \mathrm{mK}$. However, the thermal conductivity of CNF/FeG-30 is slight changed $(9.7 \mathrm{~W} / \mathrm{mK})$, compared with that of CNF/FeG-10. With the further increasing FeG content to $50 \mathrm{wt} \%$, the thermal conductivity is sharply enhanced to $30.2 \mathrm{~W} / \mathrm{mK}$, showing the enhancement of $1160 \%$ in comparison with that of pure CNF, due to the complete thermal conductive paths built by the connection of FeG sheets. Considering the tiny alteration in $\rho$ and $\mathrm{C}_{\mathrm{P}}$ of $\mathrm{CNF} / \mathrm{FeG}$ composites, the changing trend of thermal diffusivity $\mathrm{k}$ is related to thermal conductivity $\mathrm{K}$. On the basis of the LFA-467 instrument, thermal diffusivity is expressed as:

$k=0.1388 \cdot L^{2} / t_{1 / 2}$

where $t_{1 / 2}$ is half of the time that it takes for the temperature to rise to the platform area and $L$ is the sample thickness. We gather essential experimental evidences (Fig. $6 \mathrm{~b}$ and d) and find that CNF/FeG-50 expands the shortest $\mathrm{t}_{1 / 2}(231 \mathrm{~ms})$ to reach the temperature platform area, suggesting the highest thermal diffusivity $\left(29.4 \mathrm{~mm}^{2} / \mathrm{s}\right)$ and thermal conductivity $(30.2 \mathrm{~W} / \mathrm{mK})$.

The heat conduction of graphene composites is enormously dependent on several key factors: (1) good exfoliation and dispersion of GE, (2) excellent oriented lamellae structures, and (3) satisfactory interface interaction (which can enhance the phonon resonance coupling). A great deal of in-plane aligned FeG with excellent exfoliation and dispersion has been identified in the developed composites. In addition, 
intense interface interaction between $\mathrm{FeG}$ and $\mathrm{CNF}$ including hydrogen bond and " $\mathrm{Fe}-\mathrm{O}$ " complex bridge binding is conductive to the construction of thermal conductive highway. A deductive mechanism related to the stack area of fillers is proposed in Fig. 7. The thermal conductivity of polymer composites are highly determined by the thermal conductivity of matrix, thermal conductivity of fillers, thermal resistance at matrix-filler interface, and thermal resistance at filler-filler interface. Pure CNF has good thermal conductivity of $2.4 \mathrm{~W} / \mathrm{mK}$, which is grater than that of most polymer matrix, due to the wonderful aligned structures formed during the vacuum filtration. When the filler loading is less than $10 \mathrm{wt} \%$, the thermal conductivity of CNF/FeG composite is mainly leaned on the thermal conductivity and loading fraction of fillers. With the increasing FeG loading, the phonon scattering in insulating matrix is largely decreased, contributing to the improved thermal conductivity. Although the insulating bulk is reduced with the increasing FeG content at the filler range of $10-30 \mathrm{wt} \%$, a large amount of FeG breaks the in-plane orientation structures of CNF. Thus, the thermal conductivity of CNF/FeG composites is slightly changed with the increasing FeG content in this region. At the FeG content higher than $30 \mathrm{wt} \%$, FeG sheets begin to contact with each other and build the thermally conducting highway. In this stage, the thermal conductivity is primely dependent on the filler-filler thermal interfacial resistance and sharply enhanced up to $30.2 \mathrm{~W} / \mathrm{mK}$.

To emphasize the advantages of CNF/FeG composites in thermal conduction and mechanical properties, we compared the developed composites with common CNF/GNP composites, which is given in Fig. 8. As above mentioned, high filler loading will cause insufficient interfacial bonding, agglomeration of fillers, and formation of air pockets, which makes the composites brittle and difficult to process. Although CNF can disperse and strip multilayer graphene to some extent (Malho 2012; Hamedi 2014; Xu 2018; Chen 2018), its effectiveness is strongly dependent on the mixing operation technology. In control experiments, CNF/GNP-50 is too brittle to fabricate the complete circle film, despite having the similar thermal conduction level (Fig. 8a and b). SEM observations are conducted to in depth investigate the difference of microstructures between CNF/FeG-50 and CNF/GNP-50 (Fig. 8c-f). The images reveal that CNF/FeG-50 is a dense and robust composite with pretty in-plane orientation structures. Nonetheless, CNF/GNO displays a coarse, loose, and disordered microstructure, giving rise to the inferior strength that cannot support tensile tests.

In order to estimate the contribution of this present investigation, the comparison of thermal conduction and mechanical properties in CNF/FeG composites with the reported values in other polymeric composites is summarized in Fig. 9. Visibly, it is quite difficult to achieve simultaneously superior thermal conductivity and mechanical properties in the case of polymer-based composites. In this effort, CNF/FeG composites display high-level thermal conductivity and mechanical properties at the same time, which surpasses most reported composites in previous literatures. The comparison results clearly illuminate that the proposed method is competitive to dramatically promote mechanical properties and thermal conductivity of CNF composites.

\section{Conclusion}


In conclusion, a creative interfacial control strategy is proposed to fabricate polymeric composites with both fascinating thermal conductivity and mechanical performance. Fe ion-anchored graphene (FeG) are produced via in situ reduction of $\mathrm{GO}$ with $\mathrm{Fe} / \mathrm{Fe}^{2+}{ }^{2}$, followed by being incorporated into CNF matrix by a vacuum filtration method. The Raman and FTIR spectra clearly reveal that FeG is effectively reduced, and Fe ions are tightly anchored on FeG sheets via strong "Fe-O" complex bonds. FeG shows the wonderful exfoliation and dispersion with the help of CNF in aqueous solution, which is attributed to the intense interaction between FeG and CNF. Moreover, CNF/FeG composites have pretty in-plane orientation structures under the vacuum filtration. At FeG content of $50 \mathrm{wt} \%$, the thermal conductivity is sharply enhanced to $30.2 \mathrm{~W} / \mathrm{mK}$, showing the enhancement of $1160 \%$ in comparison with that of pure CNF, due to the complete thermal conductive paths built by the connection of FeG sheets. In addition, CNF/FeG-50 exhibits the exceptionally simultaneous increment of tensile strength (137\%), elongation at break (33\%), and Young's modulus (121\%), in contrast with pure CNF. Hence, this strategy provides a valuable insight into the design and construction of high-thermal-conductivity and high-performance polymer composites for versatile application in modern electronic devices.

\section{Declarations}

Conflicts of interest We have no conflicts of interest to declare that are relevant to the content of this article. We certify that they have no affiliations with or involvement in any organization or entity with any financial interest or non-financial interest in the subject matter or materials discussed in this manuscript. We have no financial or proprietary interests in any material discussed in this article.

Human and animal rights This article does not contain any studies with human participants or animals performed by any of the authors. All the experiments were undertaken in this study comply with the current laws of the country where they were performed.

Acknowledgements This work was supported by the National Natural Science Foundation of China (NSFC No. 51663003); Science and Technology Foundation of Guizhou Province (Grant No. [2019] 2166).

Data availability All data generated or analyzed during this study are included in this published article.

\section{References}

1. Balandin AA, Ghosh S, Bao W, Calizo I, Teweldebrhan D, Miao F, Lau CN (2008) Superior thermal conductivity of single-layer graphene. Nano. lett. 8:902-907. https://doi.org/10.1021/nl0731872.

2. Chen G, Chen T, Hou K, Ma W, Tebyetekerwa M, Cheng Y, Weng W, Zhu M (2018) Robust, hydrophilic graphene/cellulose nanocrystal fiber-based electrode with high capacitive performance and conductivity. Carbon 127:218-227. https://doi.org/10.1016/j.carbon.2017.11.012.

3. Chen J, Huang X, Zhu Y, Jiang P (2017) Cellulose nanofiber supported 3D interconnected BN nanosheets for epoxy nanocomposites with ultrahigh thermal management capability. Adv. Funct. Mater. 27:1604754. https://doi.org/10.1002/adfm.201604754. 
4. Chen X, Lim JSK, Yan W, Guo F, Liang YN, Chen H, Lambourne A, Hu X (2020) Salt Template Assisted BN Scaffold Fabrication toward Highly Thermally Conductive Epoxy Composites. ACS Appl. Mater. Interfaces 12:16987-16996. https://doi.org/10.1021/acsami.0c04882.

5. Cui P, Lee J, Hwang E, Lee H (2011) One-pot reduction of graphene oxide at subzero temperatures. Chem. Commun. 47:12370-12372. https://doi.org/10.1039/C1CC15569E.

6. Cui S, Song N, Shi L, Ding P (2020) Enhanced Thermal Conductivity of Bioinspired Nanofibrillated Cellulose Hybrid Films Based on Graphene Sheets and Nanodiamonds. ACS Sustain. Chem. Eng. 8:6363-6370. https://doi.org/10.1021/acssuschemeng.0c00420.

7. Dong L, Chen Z, Zhao X, Ma J, Lin S, Li M, Bao Y, Chu L, Leng K, Lu H (2018) A non-dispersion strategy for large-scale production of ultra-high concentration graphene slurries in water. Nat. Commun. 9:1-8. https://doi.org/10.1038/s41467-017-02580-3.

8. Duan H, Zhu H, Gao J, Yan D, Dai K, Yang Y, Zhao G, Liu Y, Li Z (2020) Asymmetric conductive polymer composite foam for absorption dominated ultra-efficient electromagnetic interference shielding with extremely low reflection characteristics. J. Mater. Chem. A 8:9146-9159. https://doi.org/10.1039/D0TA01393E.

9. Fan ZJ, Kai W, Yan J, Wei T, Zhi LJ, Feng J, Ren YM, Song LP, Wei F (2011) Facile synthesis of graphene nanosheets via Fe reduction of exfoliated graphite oxide. ACS Nano 5:191-198. https://doi.org/10.1021/nn102339t.

10. Ferrari AC, Robertson J (2000) Interpretation of Raman spectra of disordered and amorphous carbon. Phys. Rev. B 61:14095. https://doi.org/10.1103/PhysRevB.61.14095.

11. Geng X, Guo Y, Li D, Li W, Zhu C, Wei X, Chen M, Gao S, Qiu S, Gong Y (2013) Interlayer catalytic exfoliation realizing scalable production of large-size pristine few-layer graphene. Sci. Rep. 3:1-6. https://doi.org/10.1038/srep01134.

12. Gong S, Zhang Q, Wang R, Jiang L, Cheng Q (2017) Synergistically toughening nacre-like graphene nanocomposites via gel-film transformation. J. Mater. Chem. A 5:16386-16392. https://doi.org/10.1039/C7TA03535G.

13. Guo Y, Yang X, Ruan K, Kong J, Dong M, Zhang J, Gu J, Guo Z (2019) Reduced graphene oxide heterostructured silver nanoparticles significantly enhanced thermal conductivities in hot-pressed electrospun polyimide nanocomposites. ACS Appl. Mater. Interfaces 11:25465-25473. https://doi.org/10.1021/acsami.9b10161.

14. Hamedi MM, Hajian A, Fall AB, Håkansson K, Salajkova M, Lundell F, Wågberg L, Berglund LA (2014) Highly Conducting, Strong Nanocomposites Based on Nanocellulose-Assisted Aqueous Dispersions of Single-Wall Carbon Nanotubes. ACS Nano 8:2467-76. https://doi.org/10.1021/nn4060368.

15. Han J, Du G, Gao W, Bai H (2019) An anisotropically high thermal conductive boron nitride/epoxy composite based on nacre-mimetic 3D network. Adv. Funct. Mater. 29:1900412. https://doi.org/10.1002/adfm.201900412.

16. He H, Klinowski J, Forster M, Lerf A (1998) A new structural model for graphite oxide. Chem. Phys. Lett. 287:53-56. https://doi.org/10.1016/S0009-2614(98)00144-4. 
17. Hu J, Huang Y, Yao Y, Pan G, Sun J, Zeng X, Sun R, Xu JB, Song B, Wong CP (2017) Polymer composite with improved thermal conductivity by constructing a hierarchically ordered threedimensional interconnected network of BN. ACS Appl. Mater. Interfaces 9:13544-13553. https://doi.org/10.1021/acsami.7b02410.

18. Jha PK, Singh SK, Kumar V, Rana S, Kurungot S, Ballav N (2017) High-level supercapacitive performance of chemically reduced graphene oxide. Chem 3:846-860. https://doi.org/10.1016/j.chempr.2017.08.011.

19. Jiang F, Li T, Li Y, Zhang Y, Gong A, Dai J, Hitz E, Luo W, Hu L (2018) Wood-based nanotechnologies toward sustainability. Adv. Mater. 30:1703453. https://doi.org/10.1002/adma.201703453.

20. Kemp W (2017) Organic spectroscopy. Macmillan International Higher Education. https://doi.org/10.1007/978-1-349-15203-2.

21. Lerf A, He H, Forster M, Klinowski J (1998) Structure of graphite oxide revisited. J. Phys. Chem. B 102:4477-4482. https://doi.org/10.1021/jp9731821.

22. Li G, Tian X, Xu X, Zhou C, Wu J, Li Q, Zhang L, Yang F, Li Y (2017) Fabrication of robust and highly thermally conductive nanofibrillated cellulose/graphite nanoplatelets composite papers. Compos. Sci. Technol. 138:179-185. https://doi.org/10.1016/j.compscitech.2016.12.001.

23. Li J, Zhao X, Wu W, Zhang Z, Xian Y, Lin Y, Lu Y, Zhang L (2020) Advanced flexible rGO-BN natural rubber films with high thermal conductivity for improved thermal management capability. Carbon 162:46-55. https://doi.org/10.1016/j.carbon.2020.02.012.

24. Lu J, Askeland P, Drzal LT (2008) Surface modification of microfibrillated cellulose for epoxy composite applications. Polymer 49:1285-1296. https://doi.org/10.1016/j.polymer.2008.01.028.

25. Luo L, Wu K, Shi J, Du X, Li X, Yang L, Lu M (2017) Green reduction of graphene oxide by polydopamine to a construct flexible film: superior flame retardancy and high thermal conductivity. J. Mater. Chem. A 5:18542-18550. https://doi.org/10.1039/C7TA04740A.

26. Ma T, Zhao Y, Ruan K, Liu X, Zhang J, Guo Y, Yang X, Kong J, Gu J (2019) Highly thermal conductivities, excellent mechanical robustness and flexibility, and outstanding thermal stabilities of aramid nanofiber composite papers with nacre-mimetic layered structures. ACS Appl. Mater. Interfaces 12:1677-1686. https://doi.org/10.1021/acsami.9b19844.

27. Malho JM, Laaksonen PI, Walther A, Ikkala O, Linder MB (2012) Facile Method for Stiff, Tough, and Strong Nanocomposites by Direct Exfoliation of Multilayered Graphene into Native Nanocellulose Matrix. Biomacromolecules 13:1093. https://doi.org/10.1021/bm2018189.

28. Meng X, Pan H, Zhu C, Chen Z, Lu T, Xu D, Li Y, Zhu S (2018) Coupled chiral structure in graphenebased film for ultrahigh thermal conductivity in both in-plane and through-plane directions. ACS Appl. Mater. Interfaces 10:22611-22622. https://doi.org/10.1021/acsami.8b05514.

29. Ni Z, Wang Y, Yu T, Shen Z (2008) Raman spectroscopy and imaging of graphene. Nano Res. 1:273291. https://doi.org/10.1007/s12274-008-8036-1.

30. Park S, Lee KS, Bozoklu G, Cai W, Nguyen ST, Ruoff RS (2008) Graphene oxide papers modified by divalent ions-enhancing mechanical properties via chemical cross-linking. ACS Nano 2:572-578. 
https://doi.org/10.1021/nn700349a.

31. Park W, Hu J, Jauregui LA, Ruan X, Chen YP (2014) Electrical and thermal conductivities of reduced graphene oxide/polystyrene composites. Appl. Phys. Lett. 104:113101. https://doi.org/10.1063/1.4869026.

32. Ramanathan T, Abdala A, Stankovich S, Dikin D, Herrera-Alonso M, Piner R, Adamson D, Schniepp H, Chen X, Ruoff R (2008) Functionalized graphene sheets for polymer nanocomposites. Nat. Nanotechnol. 3:327-331. https://doi.org/10.1038/nnano.2008.96.

33. Ren W, Zhu H, Yang Y, Chen Y, Duan H, Zhao G, Liu Y (2020) Flexible and robust silver coated nonwoven fabric reinforced waterborne polyurethane films for ultra-efficient electromagnetic shielding. Compos. Part. B-Eng. 184:107745. https://doi.org/10.1016/j.compositesb.2020.107745.

34. Schiffres SN, Harish S, Maruyama S, Shiomi J, Malen JA (2013) Tunable electrical and thermal transport in ice-templated multilayer graphene nanocomposites through freezing rate control. ACS Nano 7:11183-11189. https://doi.org/10.1021/nn404935m.

35. Scott Al (2013) Interpretation of the Ultraviolet Spectra of Natural Products: International Series of Monographs on Organic Chemistry. Elsevier. Vol. 7.

36. Shen X, Wang Z, Wu Y, Liu X, He YB, Kim JK (2016) Multilayer graphene enables higher efficiency in improving thermal conductivities of graphene/epoxy composites. Nano lett. 16:3585-3593. https://doi.org/10.1021/acs.nanolett.6b00722.

37. Shen Z, Feng J (2018) Highly thermally conductive composite films based on nanofibrillated cellulose in situ coated with a small amount of silver nanoparticles. ACS Appl. Mater. Interfaces 10:24193-24200. https://doi.org/10.1021/acsami.8b07249.

38. Song H, Liu J, Liu B, Wu J, Cheng HM, F. Kang (2018) Two-dimensional materials for thermal management applications. Joule 2:442-463. https://doi.org/10.1016/j.joule.2018.01.006.

39. Song N, Pan H, Liang X, Cao D, Shi L, Ding P (2018) Structural design of multilayer thermally conductive nanofibrillated cellulose hybrid film with electrically insulating and antistatic properties. J. Mater. Chem. C 6:7085-7091. https://doi.org/10.1039/C8TC01277F.

40. Stankovich S, Dikin DA, Dommett GH, Kohlhaas KM, Zimney EJ, Stach EA, Piner RD, Nguyen ST, Ruoff RS (2006) Graphene-based composite materials. Nature 442:282-286. https://doi.org/10.1038/nature04969.

41. Tian S, Sun J, Yang S, He P, Wang G, Di Z, Ding G, Xie X, Jiang M (2016) Controllable edge oxidation and bubbling exfoliation enable the fabrication of high quality water dispersible graphene. Sci. Rep. 6:34127. https://doi.org/10.1038/srep34127.

42. Uetani K, Okada T, Oyama HT (2016) Thermally conductive and optically transparent flexible films with surface-exposed nanocellulose skeletons. J. Mater. Chem. C 4:9697-9703. https://doi.org/10.1039/C6TC03318K.

43. Wan S, Fang S, Jiang L, Cheng Q, Baughman RH (2018) Strong, conductive, foldable graphene sheets by sequential ionic and $\pi$ bridging. Adv. Mater. 30:1802733. https://doi.org/10.1002/adma.201802733. 
44. Wu D, Gao X, Sun J, Wu D, Liu Y, Kormakov S, Zheng X, Wu L, Huang Y, Guo Z (2017) Spatial Confining Forced Network Assembly for preparation of high-performance conductive polymeric composites. Compos. Part A-Appl. S 102:88-95. https://doi.org/10.1016/j.compositesa.2017.07.027.

45. Wu Z, Xu C, Ma C, Liu Z, Cheng HM, Ren W (2019) Synergistic effect of aligned graphene nanosheets in graphene foam for high-performance thermally conductive composites. Adv. Mater. 31:1900199. https://doi.org/10.1002/adma.201900199.

46. Xu X, Chen J, Zhou J, Li B (2018) Thermal conductivity of polymers and their nanocomposites. Adv. Mater. 30:1705544. https://doi.org/10.1002/adma.201705544.

47. Xu Y, Sheng K, Li C, Shi G (2010) Self-assembled graphene hydrogel via a one-step hydrothermal process. ACS Nano 4:4324-4330. https://doi.org/10.1021/nn101187z.

48. Xu Y, Yang Y, Yan D, Duan H, Zhao G, Liu Y (2018) Gradient structure design of flexible waterborne polyurethane conductive films for ultraefficient electrimagnetic shielding with low reflection characteristic. ACS Appl. Mater. Interfaces 10:19143-19152. https://doi.org/10.1021/acsami.8b05129.

49. Yang W, Gong Y, Zhao X, Liu T, Zhang Y, Chen F, Fu Q (2019) Strong and highly conductive graphene composite film based on the nanocellulose-assisted dispersion of expanded graphite and incorporation of poly (ethylene oxide). ACS Sustain. Chem. Eng. 7:5045-5056. https://doi.org/10.1021/acssuschemeng.8b05850.

50. Yang W, Zhao Z, Wu K, Huang R, Liu T, Jiang H, Chen F, Fu Q (2017) Ultrathin flexible reduced graphene oxide/cellulose nanofiber composite films with strongly anisotropic thermal conductivity and efficient electromagnetic interference shielding. J. Mater. Chem. C 5:3748-3756. https://doi.org/10.1039/C7TC00400A.

51. Yao Y, Zeng X, Wang F, Sun R, Xu JB, Wong CP (2016) Significant enhancement of thermal conductivity in bioinspired freestanding boron nitride papers filled with graphene oxide. Chem. Mater. 28:1049-1057. https://doi.org/10.1021/acs.chemmater.5b04187.

52. Yao Y, Zhu X, Zeng X, Sun R, Xu JB, Wong CP (2018) Vertically aligned and interconnected SiC nanowire networks leading to significantly enhanced thermal conductivity of polymer composites. ACS Appl. Mater. Interfaces 10:9669-9678. https://doi.org/10.1021/acsami.8b00328.

53. Yuan W, Zhou Y, Li Y, Li C, Peng H, Zhang J, Liu Z, Dai L, Shi G (2013) The edge-and basal-planespecific electrochemistry of a single-layer graphene sheet. Sci. Rep. 3:2248. https://doi.org/10.1038/srep02248.

54. Zhang Y, Peng J, Li M, Saiz E, Wolf SE, Cheng Q (2018) Bioinspired supertough graphene fiber through sequential interfacial interactions. ACS Nano 12:8901-8908. https://doi.org/10.1021/acsnano.8b04322.

55. Zhao L, Roh K, Kacmoli S, Kurdi KA, Jhulki S, Barlow S, Marder SR, Gmachl C, Rand BP (2020) Thermal Management Enables Bright and Stable Perovskite Light-Emitting Diodes. Adv. Mater. 32:2000752. https://doi.org/10.1002/adma.202000752. 
56. Zhao X, Gao W, Yao W, Jiang Y, Xu Z, Gao C (2017) lon diffusion-directed assembly approach to ultrafast coating of graphene oxide thick multilayers. ACS Nano 11:9663-9670. https://doi.org/10.1021/acsnano.7b03480.

57. Zhuo H, Zhang H, Wang L, Lu Q, Kaplan DL (2018) Sonication exfoliation of defect-free graphene in aqueous silk nanofiber solutions. ACS Sustain. Chem. Eng. 6:12261-12267. https://doi.org/10.1021/acssuschemeng.8b02644.

\section{Figures}

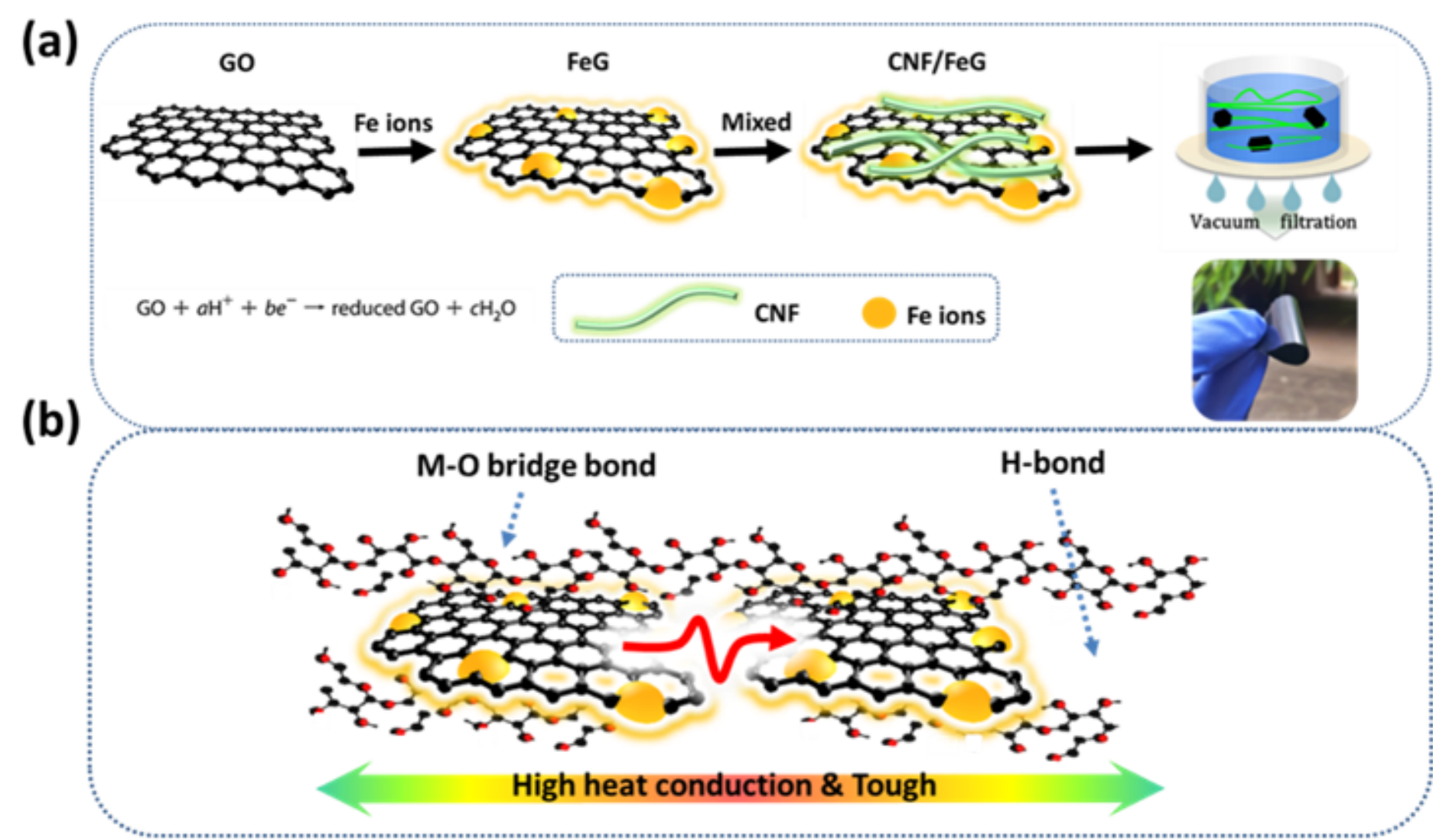

(c)

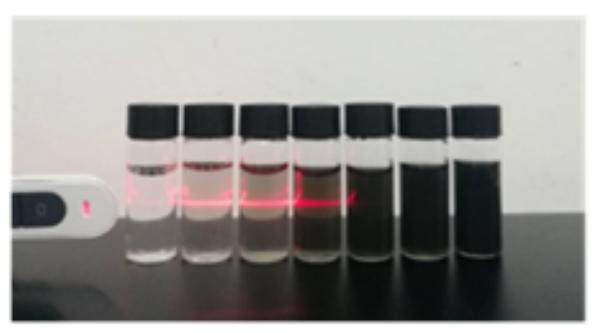

(d)

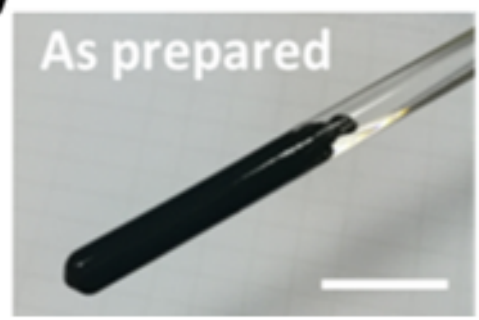

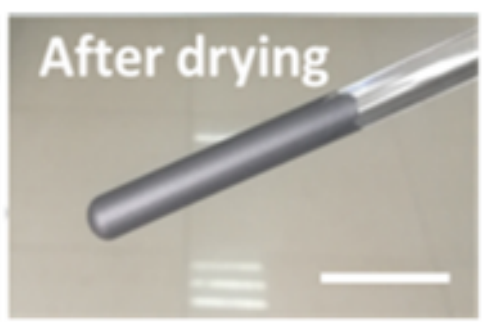

Figure 1

(a) Schematic diagram of the preparation process of FeG and CNF/FeG composites, (b) Schematic of the bridge binding between $\mathrm{CNF}$ and FeG, (c) Optical photo of CNF/FeG aqueous suspensions, (d) Optical photos for FeG coating on a glass rod 
(a)

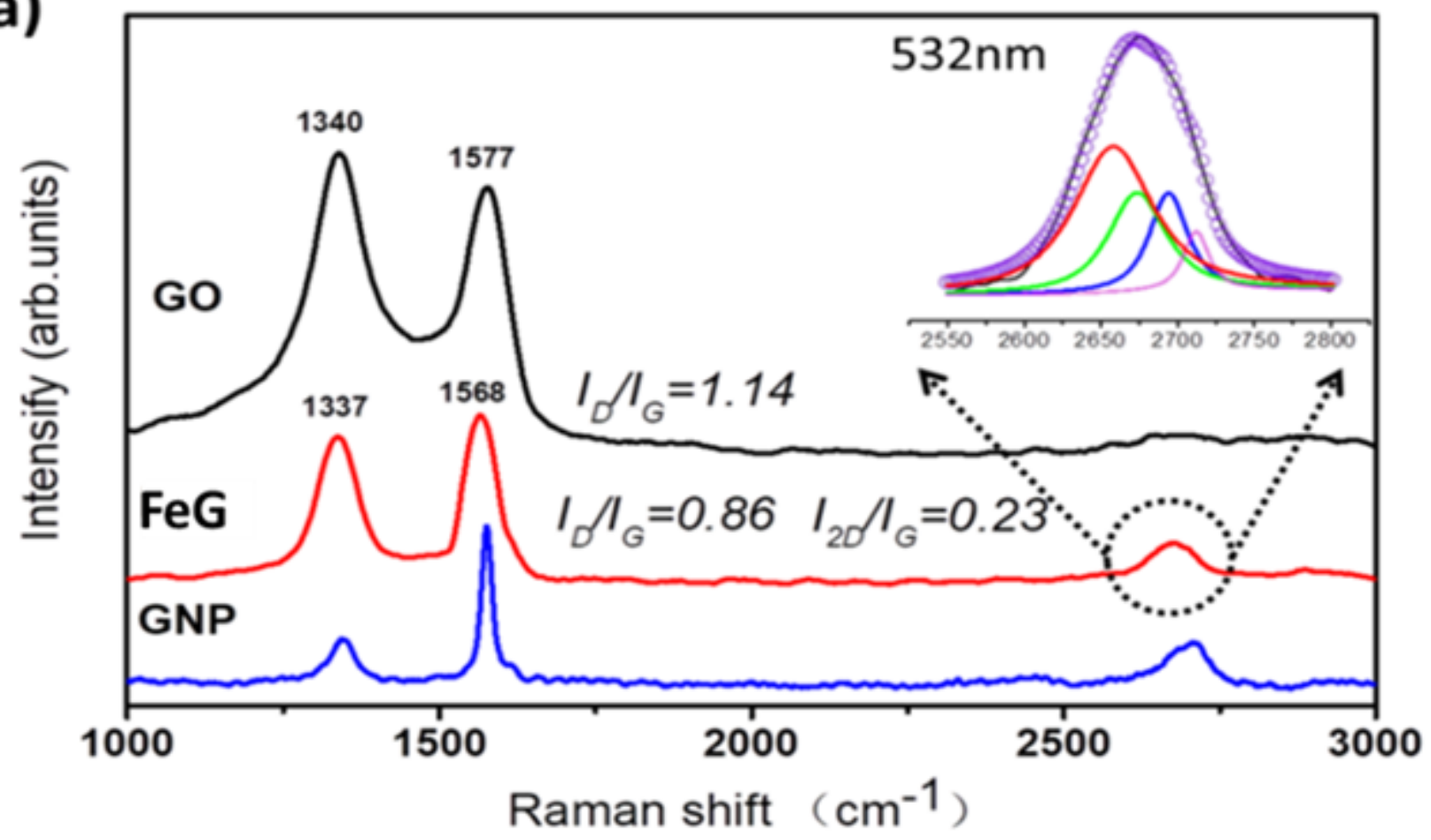

(b)

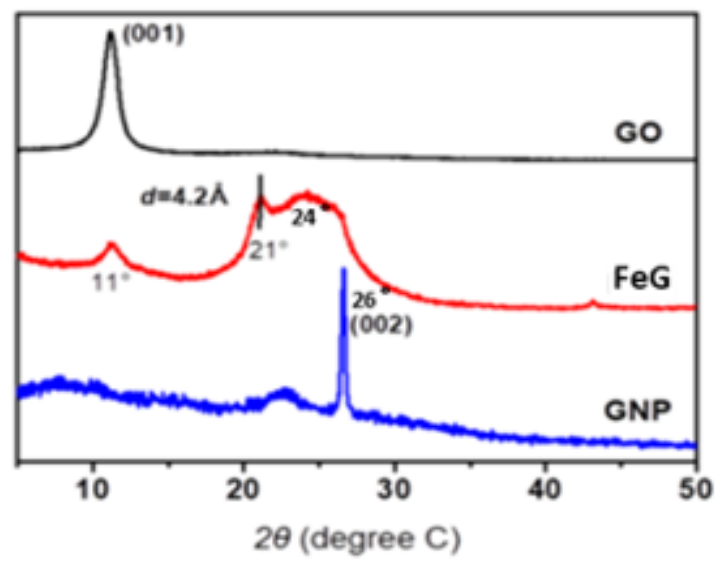

(c)

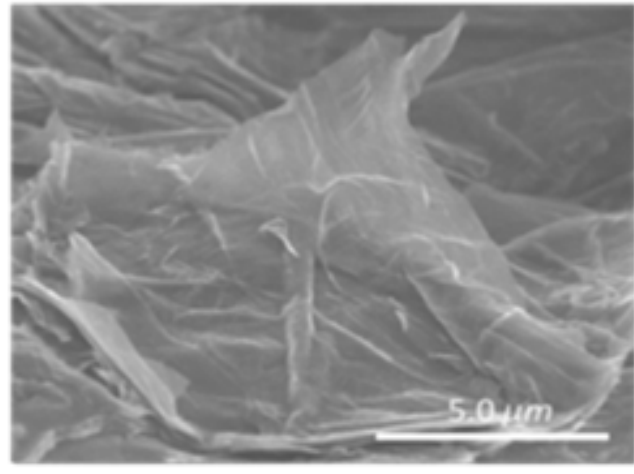

Figure 2

(a) Raman spectra and (b) XRD patterns of GO, FeG and GNP, (c) SEM image of FeG 
(a)

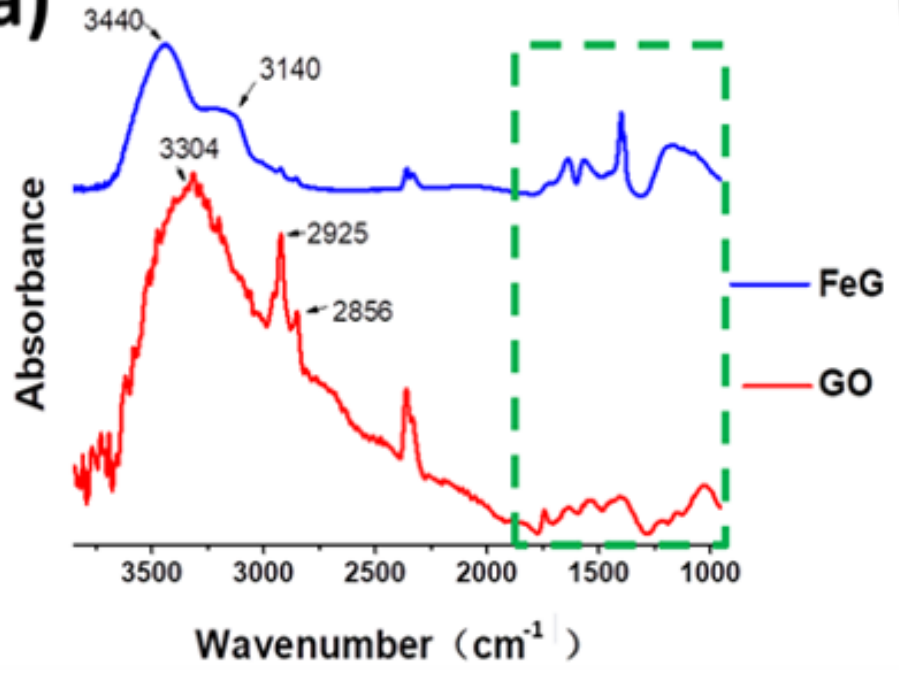

(b)

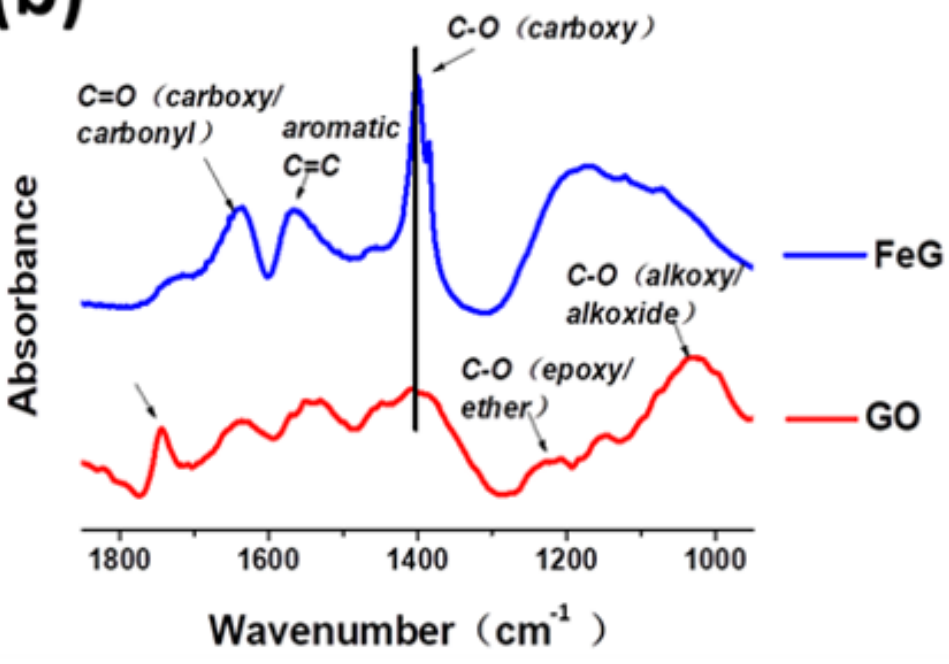

Figure 3

FTIR spectra of FeG and GO in (a) 4000-1000 cm-1 and (b) $1800-1000 \mathrm{~cm}-1$ 

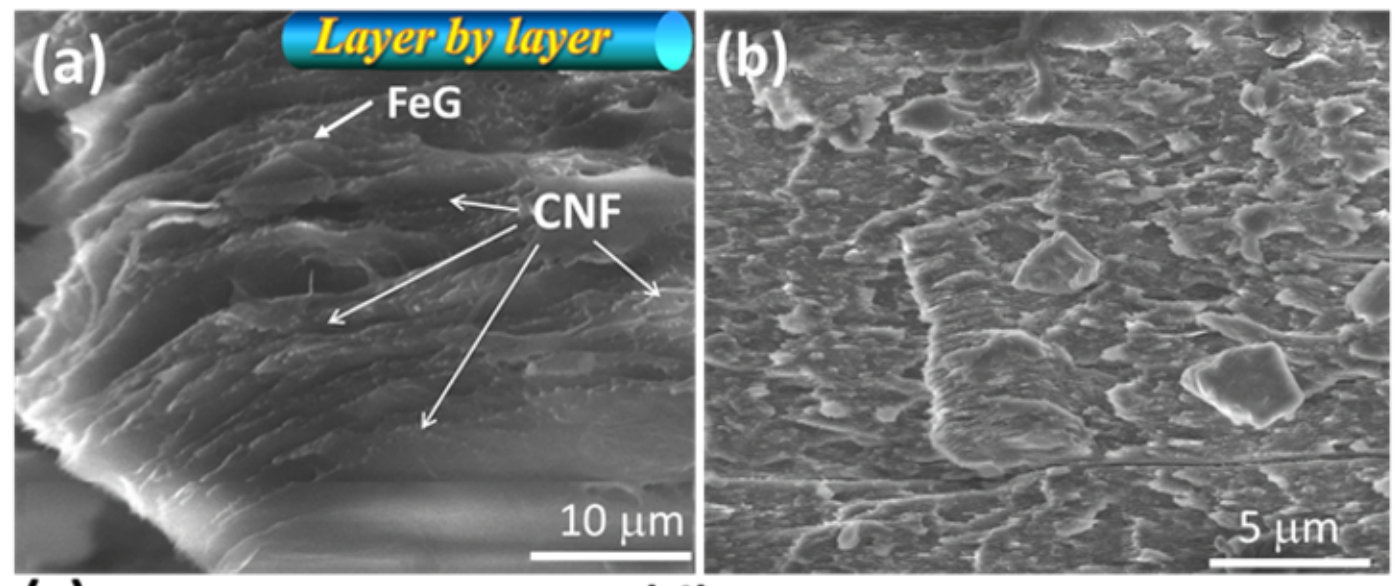

\section{(c)}

(d)

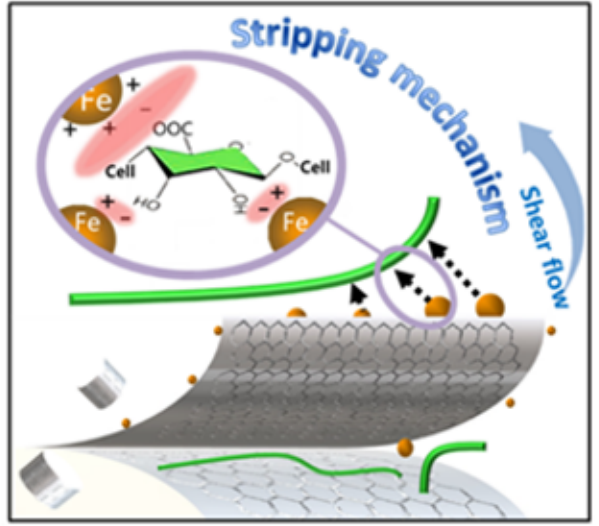

(e)

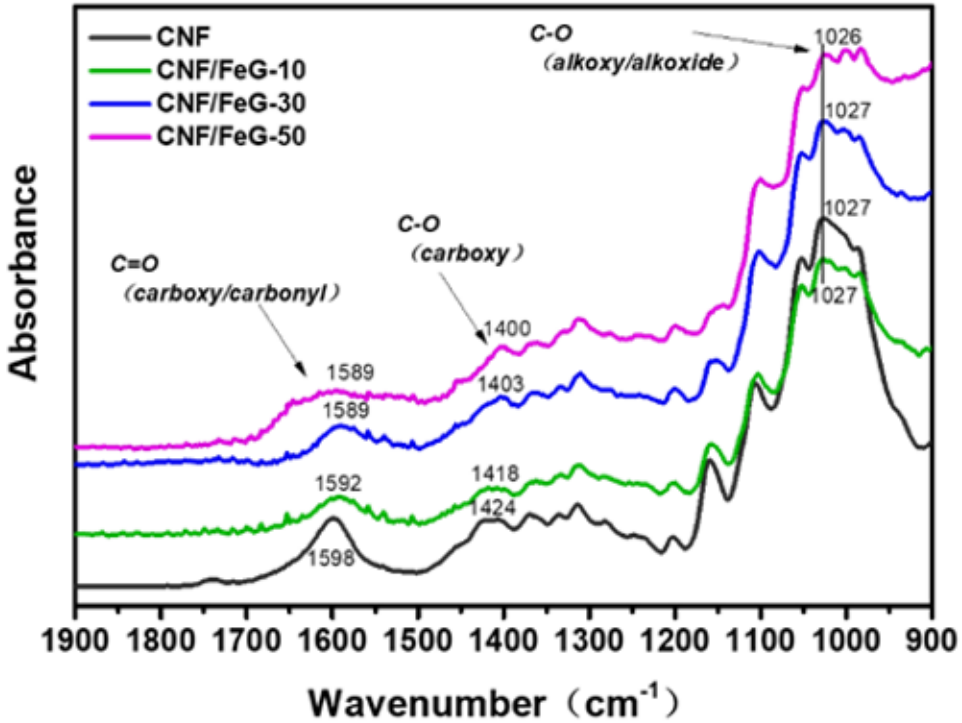

(f)

\section{CNF solution CNF/FeG solution}
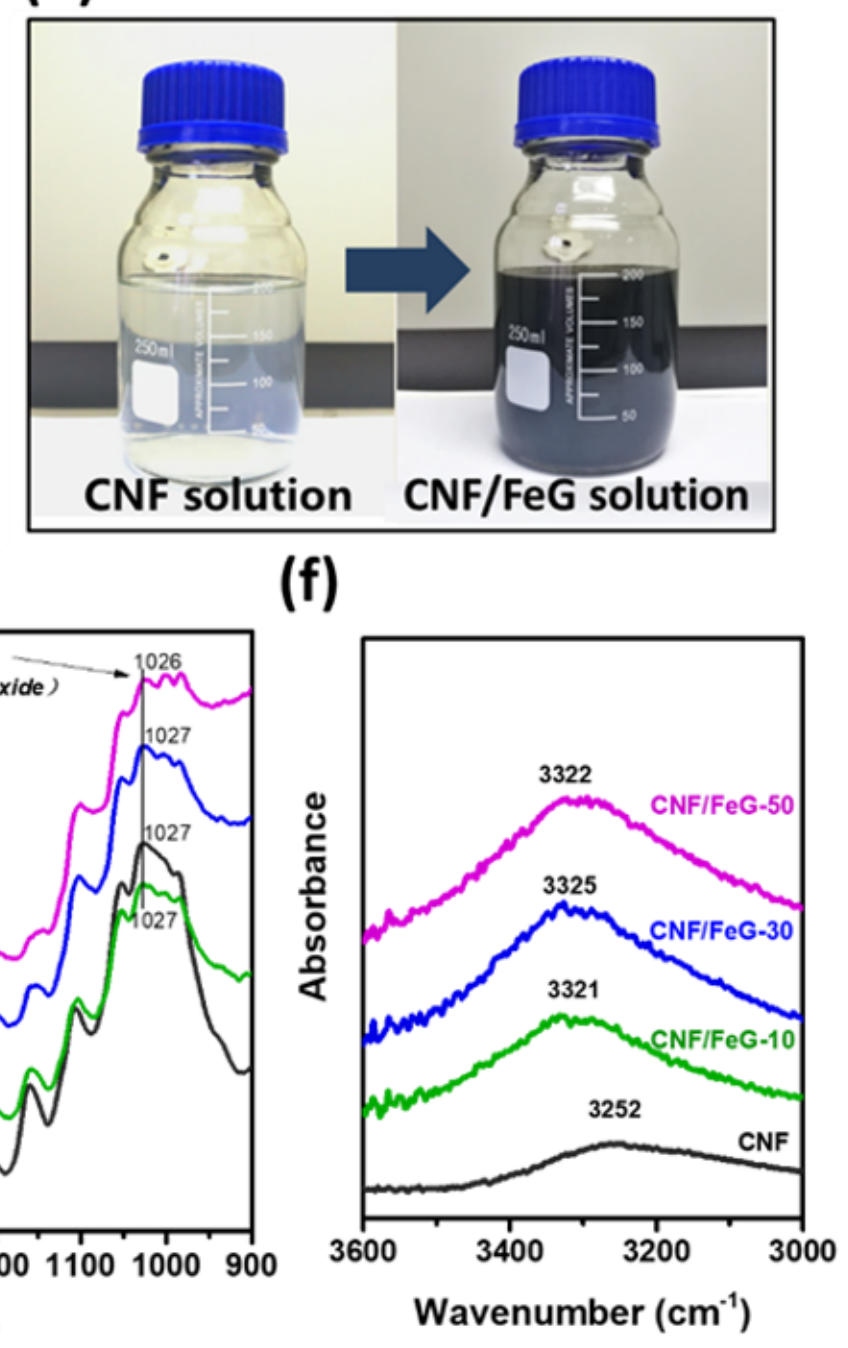

Figure 4

(a, b) Cross-section SEM characterizations of CNF/FeG-50 composite, (c) Schematic diagram of CNF/FeG stripping and dispersion mechanism, (d) Digital pictures of CNF and CNF/FeG aqueous dispersions, FTIR spectra of pure CNF and CNF/FeG composites in the wavenumber of (e) 1900-900 cm-1 and 3600-3000 $\mathrm{cm}-1$ (f) 
(a)

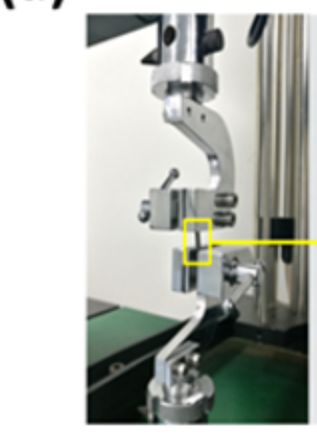

(d)

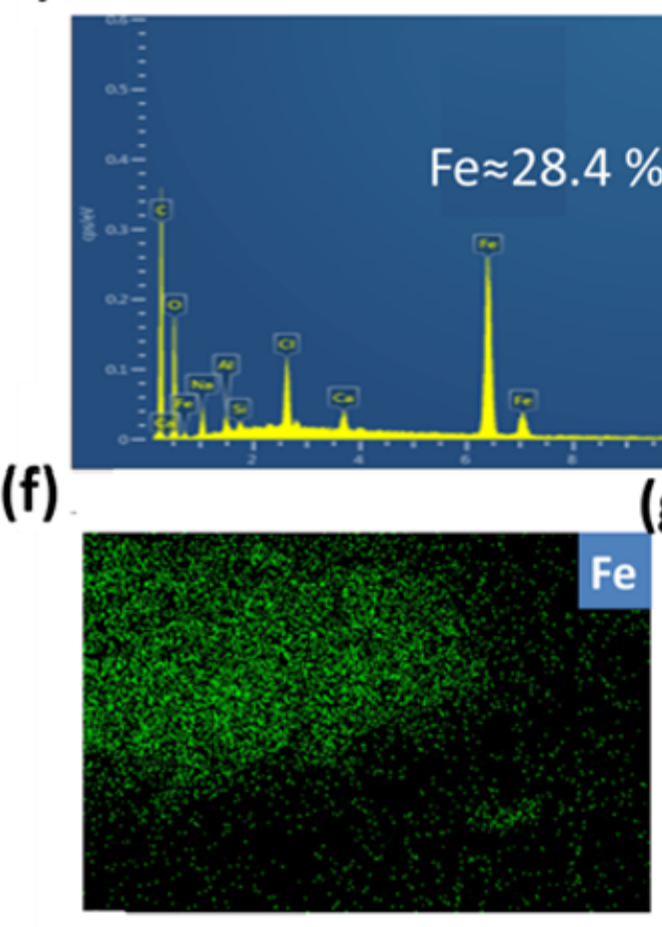

(b)

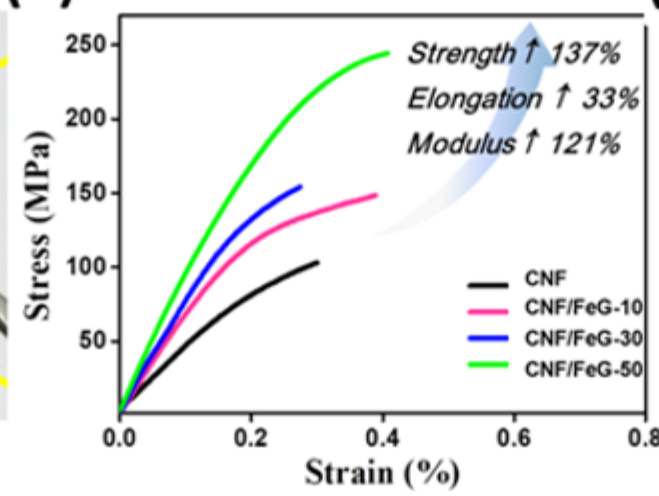

(c)

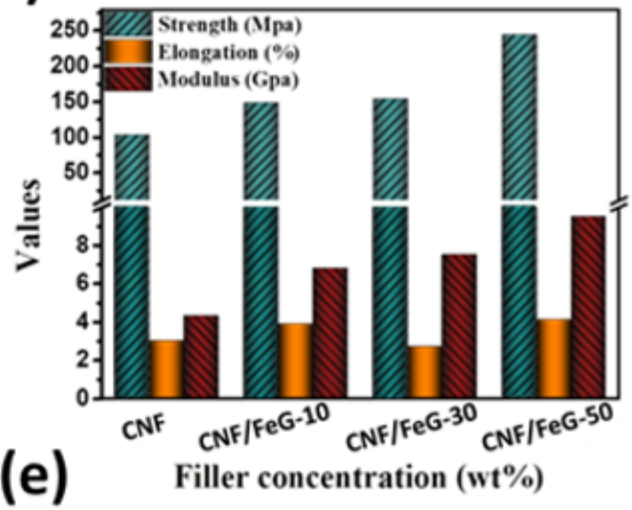

(e)

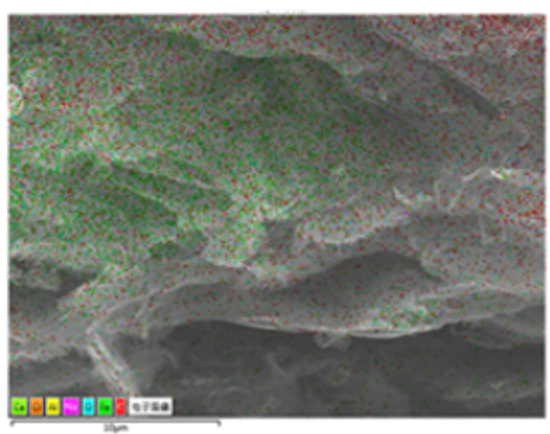

(h)
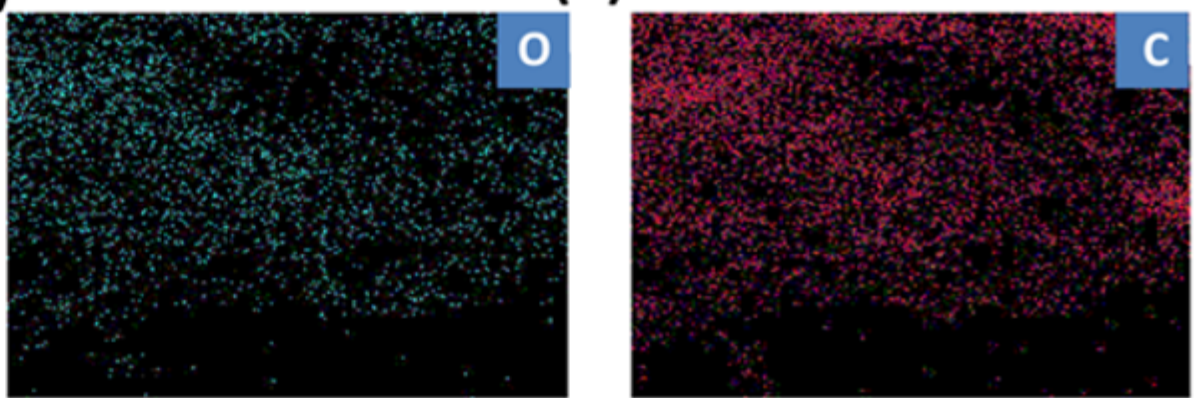

Figure 5

(a) photos of mechanical tensile tester and CNF/FeG composite, (b) Typical stress-train curves of pure CNF and CNF/FeG composites, (c) The corresponding strength, elongation, and modulus, (d) the EDS line scanning spectrum of CNF/FeG-50, (e-h) EDS mapping images of CNF/FeG-50 

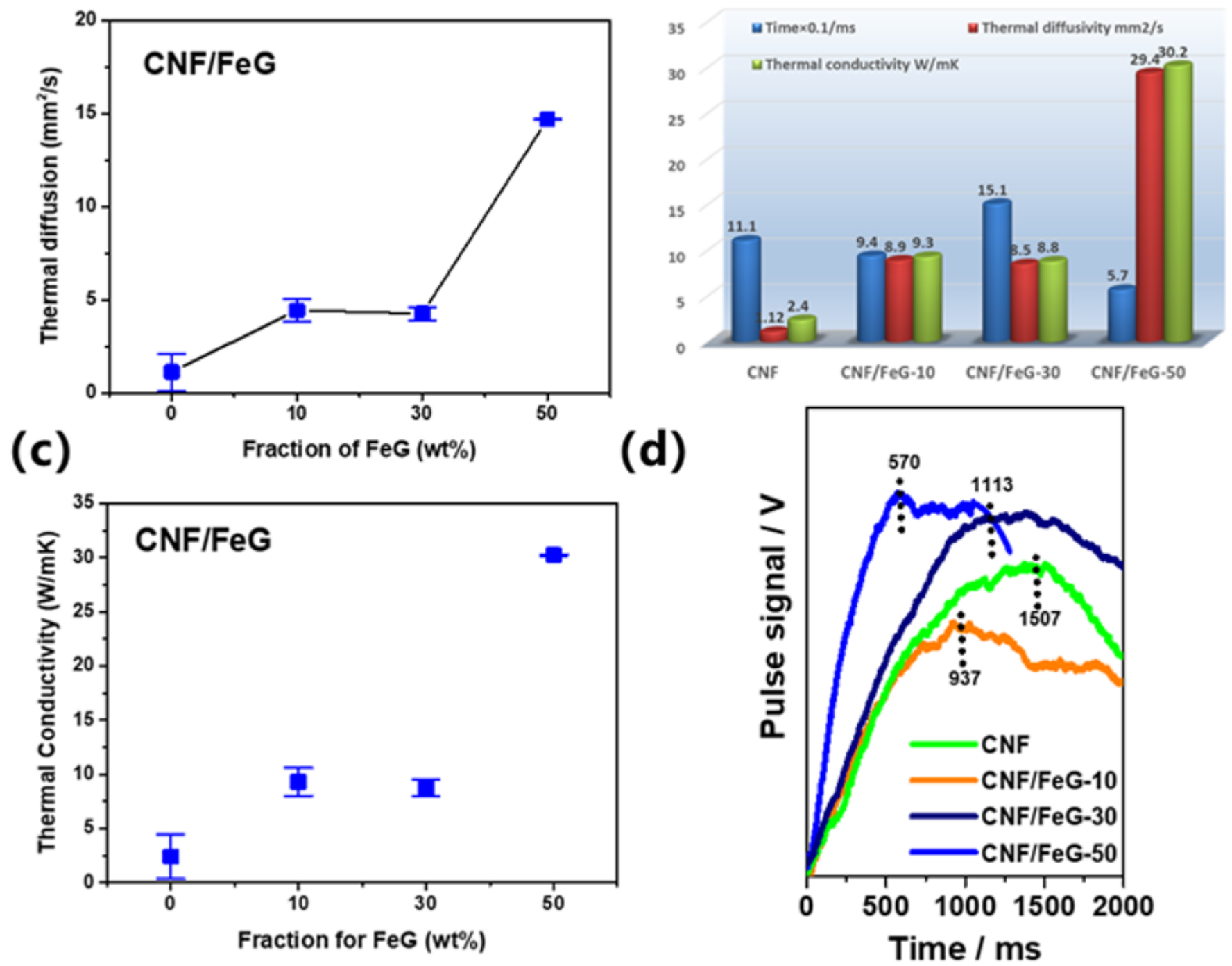

Figure 6

(a) Thermal diffusivity and (c) thermal conductivity of CNF/FeG composites with different FeG contents,

(b) The relative comparison of pulse laser response time, thermal diffusivity, and thermal conductivity for CNF/FeG composites, (d) The normalized Pulse signal-Time patterns for FeG/CNF composites with different contents 

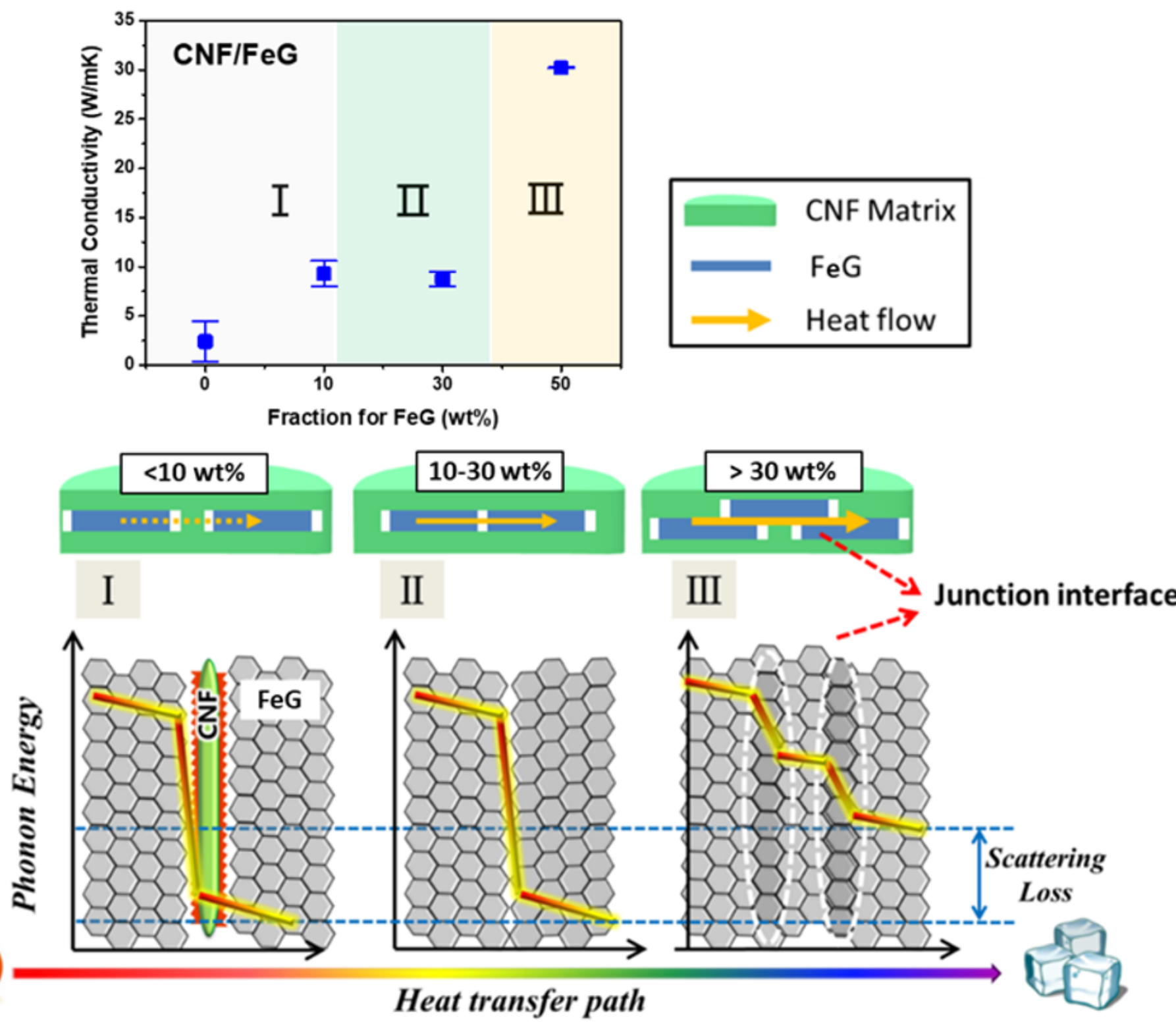

Figure 7

Schematic diagram for the thermal conduction mechanism of CNF/FeG 


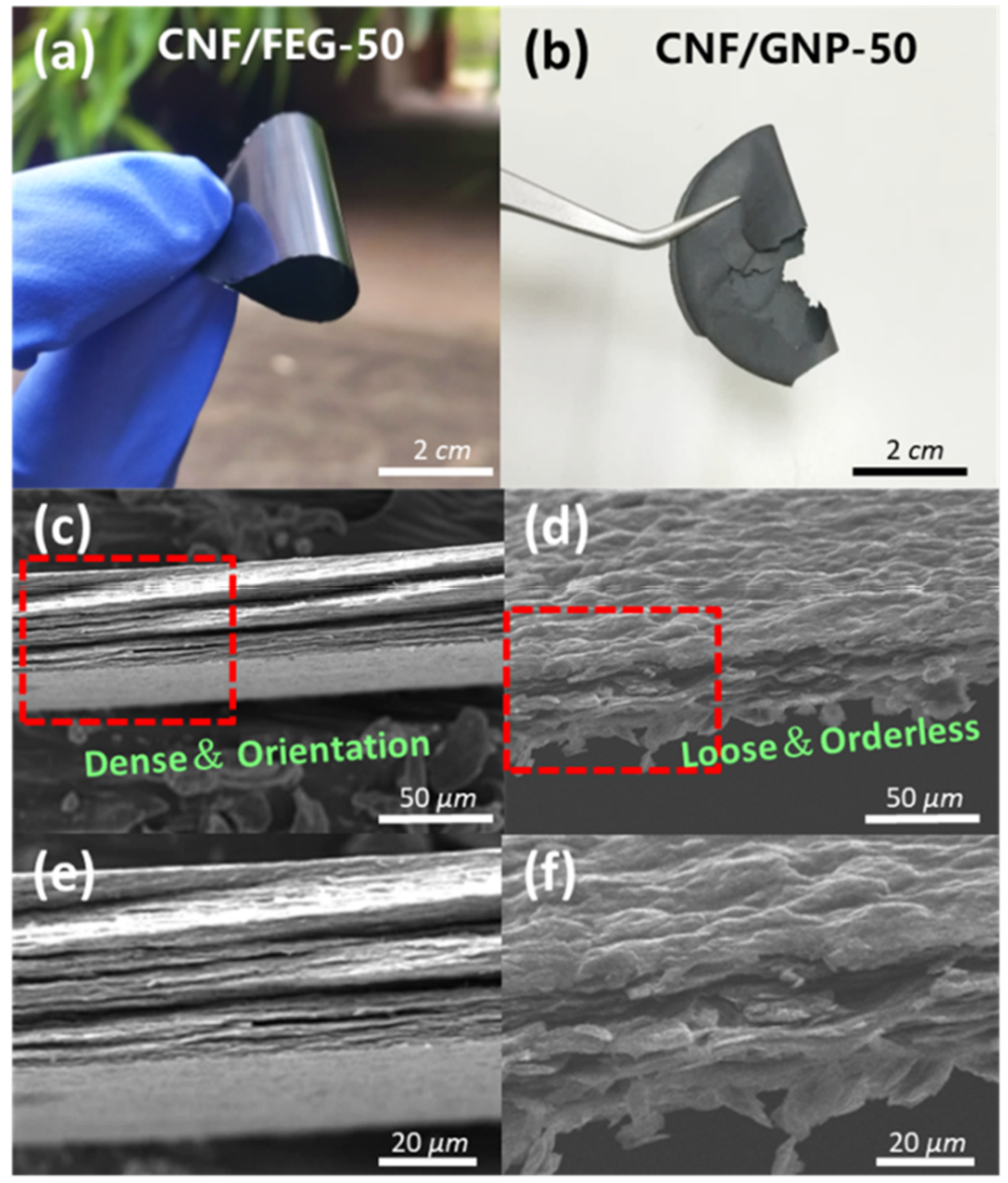

Figure 8

(a, b) Comparison of optical photos of CNF/FeG-50 and CNF/GNP-50 composites, (c, d) Cross-sectional SEM images of CNF/FeG-50 and CNF/GNP-50, respectively, $(e, f)$ Partial enlarged SEM images in (c and d) 


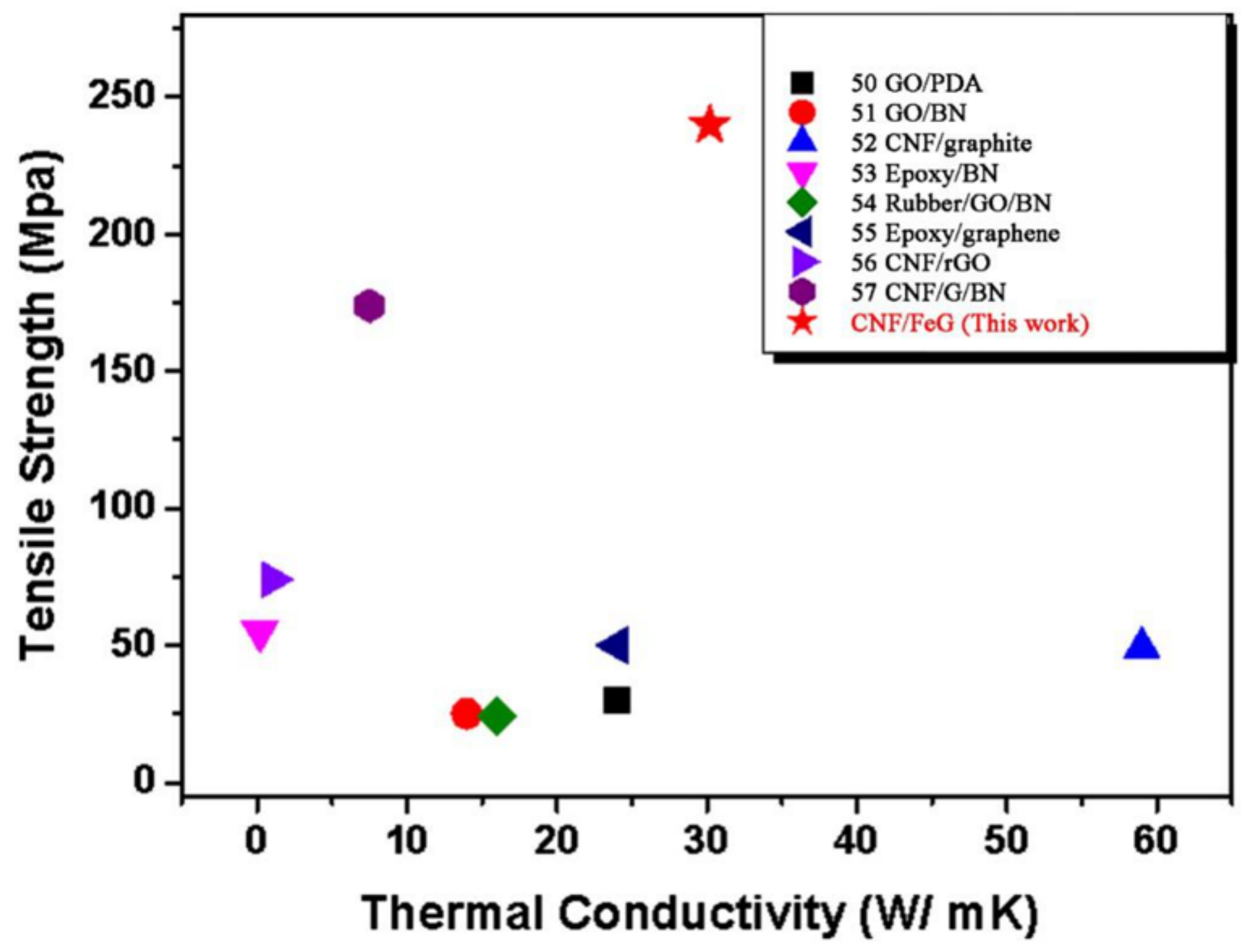

Figure 9

Comparison of thermal conductivity and tensile strength for different polymer-based composites (Luo 2017; Yao 2016; Li 2017; Chen 2020; Li 2020; Shen 2016; Yang 2017; Song 2018)

\section{Supplementary Files}

This is a list of supplementary files associated with this preprint. Click to download.

- Graphicabstract.tif 